\title{
Designing a sandwich aircraft spoiler with lattice structure cores
}

\author{
Haifeng $\mathrm{Ou}^{1}$, Jie $\mathrm{Liu}^{1,{ }^{*}}$, Junfeng $\mathrm{He}^{1}$, Zufeng Pang ${ }^{1}$, Yonghui Zhang ${ }^{1}$, Wei Wei ${ }^{1}$, \\ Hongxin Wang ${ }^{2}$, Gang Zhao ${ }^{2}$, Guilin Wen ${ }^{1,2}$ \\ ${ }^{I}$ Center for Research on Leading Technology of Special Equipment, School of Mechanical and \\ Electric Engineering, Guangzhou University, Guangzhou 510006, P. R. China \\ ${ }^{2}$ State Key Laboratory of Advanced Design and Manufacturing for Vehicle Body, Hunan University, \\ Changsha 410082, Peoples Republic of China
}

*Correspondence and requests for materials should be addressed to J.L. (email: jliu@gzhu.edu.cn)

\begin{abstract}
:
By combing continuum topology optimization (TO) method and lattice structure technique, a sandwich aircraft spoiler with a high stiffness-to-weight ratio is designed. TO method is served to produce the shell of the aircraft spoiler and the lattice structure, used as cores, is employed to support the shell. TO problem is established as maximizing the stiffness of the structure with limited material volume. Density-based method is utilized to achieve a 0/1 solution. We then empirically replace the core of the aircraft spoiler by using 3D kagome lattice structure. Two different materials, i.e., aluminum alloy and titanium alloy, are synthetically applied to further reduce the weight and simultaneously improve the strength of the aircraft spoiler. Numerical simulations are conducted to show that the designed aircraft spoiler can meet the service environment with a reduction of its weight by approximately $80 \%$ when compared with that of the initial design model. Finally, we have fabricated the designed model with photosensitive resin by using 3D printing technique.
\end{abstract}

Keywords: aircraft spoiler; topology optimization; lattice structure; high stiffness-to-weight ratio 


\section{Introduction}

A spoiler in aircraft is an equipment aimed at intentionally reducing the lift component of an airfoil in a controlled way [1]. Considering the demanding requirements for the high stiffness-to-weight ratio in the aerospace industry, the spoiler should be designed lightweight and with high stiffness [2]. Traditionally, it is designed by using trail-and-error method, which is always time-consuming and normally too conservative. Recently developed TO methods [3-5] can overcome these drawbacks.

TO methods, severed as efficient tools, can produce various novel candidates for engineering structures. Initially, these methods play a role purely in the conceptual design process in industry, following shape optimization, size optimization, and numerical verifications. The main reason is the gap between the complexity and intricacy of the optimum solution and the manufacturing technique. However, recent years have witnessed the rapid increment of the applications of the TO methods in numerous fields since manufacturing has been carefully considered in the optimization process [6-8]. For conventional subtractive manufacturing method, e.g. machining and injection molding/casting, the main concerns are adding extra constraints to the optimization model to avoid the interior voids and undercuts and control the rib thickness [6,7]. As for recently emerged additive manufacturing (AM) method, although showing a promising perspective with the ability of reducing the geometric intricacy restrictions imposed on topology optimization by conventional manufacturing, several key problems have to be dealt with, e.g., the support structure design [8-10]. The superiority of AM is more prominent when one attempt to design and fabricate sandwich structures. Sandwich structure, constructed by attaching two thin yet stiff skins to a lightweight but thick core, is typical lightweight structure with high stiffness. Because of the use of the core, the whole structure can exhibit high mechanical performance by using normally low strength material. Various cores have been proposed, e.g., tetrahedron [11], 3D kagome [12], and pyramid [13], honeycomb [14], et al. Notice that the advantages and drawbacks of these cores are not covered in this work since we purely focus on designing a novel aircraft spoiler by employing one of them. 
By using TO method, several components of the aircraft have been designed, mainly including airframe structures and stiffener ribs for aircraft panels [2]. Using a bi-level optimization scheme, two Airbus pylons were designed by combing TO method and geometric optimization [15]. Zhu et al. [16] proposed a novel TO method to improve the stiffness and strength of the aircraft skin stretch-forming die. A Heaviside-function based directional growth topology parameterization has been developed to achieve stiffener layout designs [17], having the potential for designing stiffener ribs for aircraft panels. Krog et al. have designed aircraft wing box ribs by using TO method [18]. By tackling coupled fluid-structure problems, morphing aircraft structures were designed by using a multidisciplinary TO method [19]. In addition, multi-component design problems of aircrafts have been extensively studied by Zhang and his co-works [20-22]. Most recently, novel aircraft wings [23-25] have been produced. However, these final optimized designs are generally not able to be fabricated directly, especially for finite element-based TO method, since these designs (1) have no direct link with CAD modelling system and (2) their edges are normally needed to smooth. Recently proposed morphable moving component/void (MMC/MMV) [26-29] methods have the ability of seamlessly integrating topology optimization in CAD modeling systems, which can overcome problem (1). For problem (2) various methods, including employing higher-order finite elements or refined meshes [30-32] and using meshes adaptive strategies [33-36], have been proposed. Note that level set method can inherently produce structures with smooth edges [37,38].

Nevertheless, it is always impossible to automatically obtain the desired structure by using a method. In this study, combing TO method and the empirical design, we propose a sandwich aircraft spoiler. The design is guided by the optimum layout from TO results. The internal structures, which is very complex, are manually replaced by 3D kagome cores to improve the manufacturing and the strength of the aircraft spoiler. In addition, to further reduce the weight and improve the strength of the aircraft spoiler, aluminum alloy and titanium alloy are synthetically applied to the aircraft spoiler. We conduct the numerical simulations to investigate whether the designed aircraft spoiler can meet the service environment. Finally, a novel aircraft spoiler model is fabricated by using photosensitive resin. 


\section{Materials and methods}

\subsection{Topology optimization method}

Topology optimization aims to obtain the optimal material layout under the prescribe loading and boundary condition with the given material. Density-based method treats the material density of each element as the design variable between 0 (void element)-1 (solid element) by allowing the material to take intermediate densities (gray element). The Young's modulus of the elements in the design domain is parameterized with design variable and the properties of intermediate densities are artificially penalized [39]. In order to achieve penalized intermediate densities, the Young's modulus of the eth element can be expressed as,

$$
E_{e}=x_{e}^{p} E_{s}
$$

where $E_{s}$ is the Young's modulus of the solid element, $x_{e}$ is the relative density of material element (i.e., the design vector of the element densities), and $p$ as the penalization power is used to suppress intermediate density and ensures good convergence to 0/1 designs, which usually has a value of 3 .

Let the structure be discretized into $N$ finite elements (i.e., the number of design variables) and let $\boldsymbol{k}_{e}$ be the element stiffness matrix, then, the global stiffness matrix can be expressed as:

$$
\boldsymbol{K}=\sum_{e=1}^{N} x_{e}^{p} \boldsymbol{k}_{e}
$$

Maximizing the structural stiffness is equivalent to minimize the structural compliance, thus, the TO problem can be formulated as,

$$
\begin{aligned}
\text { Min : } & c(\mathbf{x})=\boldsymbol{U}^{T} \boldsymbol{K} \boldsymbol{U}=\sum_{e=1}^{N}\left(x_{e}\right)^{p} \boldsymbol{u}_{e}^{T} \boldsymbol{k}_{e} \boldsymbol{u}_{e} \\
\text { s.t.: } & \frac{V(\mathbf{x})}{V_{0}}=f \\
: & \boldsymbol{K} \boldsymbol{U}=\boldsymbol{F} \\
: & \mathbf{0}<\boldsymbol{x}_{\text {min }} \leq \boldsymbol{x} \leq \mathbf{1}
\end{aligned}
$$

where $c(\boldsymbol{x})$ is the objective function, $\boldsymbol{F}$ is the global load vectors, $\boldsymbol{U}$ and $\boldsymbol{u}_{e}$ are the global and element displacement vectors, respectively, $\boldsymbol{V}_{(x)}$ and $\boldsymbol{V}_{0}$ are the solid material volume and design domain volume, respectively, and $f$ is the prescribed volume fraction (i.e., the ratio of optimized 
structure to design domain), $\boldsymbol{x}_{\min }$ is a vector of minimum relative densities, which avoids non-positive definite stiffness matrix, and its value is usually set to be 0.001 .

The optimization presented in Eq. (3) can be solved by utilizing many methods, e.g., the method of moving asymptotes (MMA) [40], optimality criterion (OC) method [41], linear or sequential quadratic programming method [42-44], and ESO method [45], et al.

\subsection{Design problem definition}

The initial design domain of the aircraft spoiler is shown in Figure 1(a). The aircraft spoiler consists of a skin (highlighted by green), joints (highlighted by red), and the main structure (highlighted by red). The initial thickness of the skin is set to be $1 \mathrm{~mm}$. The skin domain has the length and width of $1220 \times 426 \mathrm{~mm}$, and is discretized by $21,551 \mathrm{~S} 4$ elements. The remaining domain has a length of $1220 \mathrm{~mm}$ and a width of $470 \mathrm{~mm}$, which is occupied by 149,779 C3D8 elements. Two non-design domains, the skin and joints, are defined to maintain the integrity of the final design. The objective function is to minimize the structural flexibility under a volume constraint of $20 \%$. $x$-direction of all joints is constrained, $y$-direction of joints 1 and 2 is constrained, and z-direction of joints 2, 3, 4, and 5 is fixed. A distributed uniform load of 20,000 $\mathrm{Pa}$ is applied at the structural upper skin along the vertical skin. Aluminum alloy and titanium alloy are used to construct the optimized aircraft spoiler. Their properties are shown in Table 1.

Table 1. Properties of the used materials

\begin{tabular}{ccc}
\hline Material & Titanium alloy & Aluminum alloy \\
\hline Density & $4500 \mathrm{Kg} / \mathrm{m}^{3}$ & $2760 \mathrm{Kg} / \mathrm{m}^{3}$ \\
Tensile strength & $900 \mathrm{MPa}$ & $450 \mathrm{MPa}$ \\
Compressive strength & $880 \mathrm{MPa}$ & $270 \mathrm{MPa}$ \\
Modulus of elasticity & $108 \mathrm{GPa}$ & $68 \mathrm{GPa}$ \\
Poisson's ratio & 0.33 & 0.33 \\
\hline
\end{tabular}

\section{Results and discussions}

Figure 1(b) shows the optimal material layout of the aircraft spoiler and the Mises equivalent stress distribution. The maximum stress (stress concentration) of this structure occurs at the joints as expected with a maximum value of $256.6 \mathrm{MPa}$. Since there is a subsequent design, the regions with material density greater than 0.4 are replaced by solid materials and those less than 0.4 are 
removed, as shown in Figure 1(c). Based on this, the optimized model in Figure 1(c) is remodeled manually in SolidWorks, as shown in Figure 2(a). The remodeled aircraft spoiler includes the skin (colored green), the lower surface (colored blue), and joints (colored gray). In addition, sharp corners are rounded to avoid the stress concentration. The internal structure, linking the skin and the lower surface, is removed to make room for the lattice structure. Note that the maximum stress occurs at the surface of the joints. Hence, we increase the thickness of the joint to be $4 \mathrm{~mm}$. Moreover, to ensure the designed structure can be easily fabricated by using a normal 3D printing machine, the thickness of both the skin and lower surface is set to be $2.5 \mathrm{~mm}$.

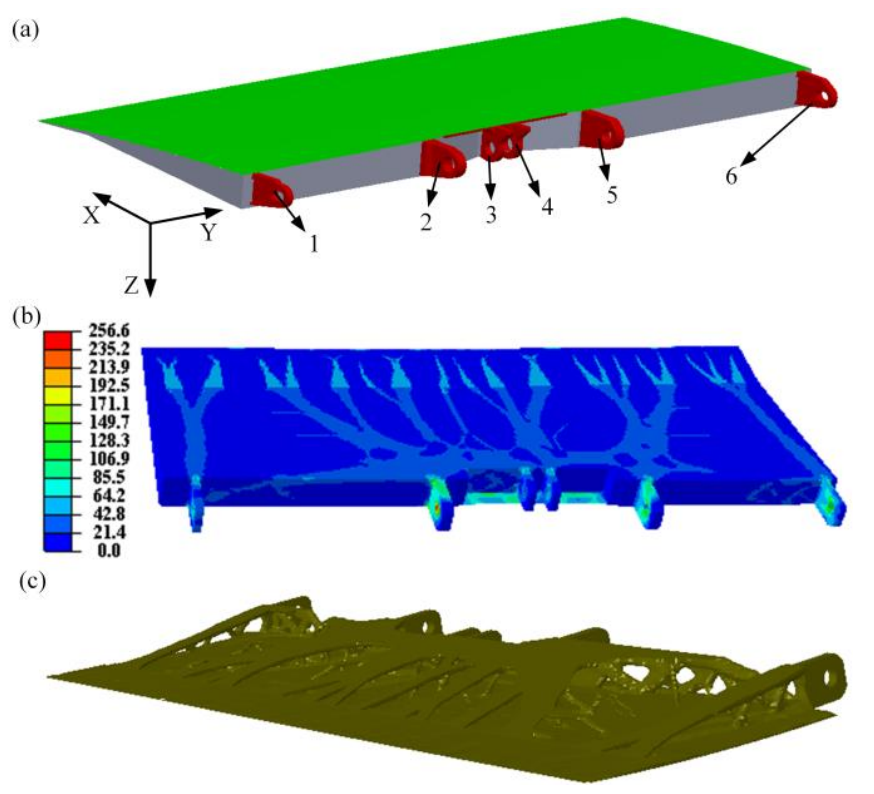

Figure 1. (a) An initial aircraft spoiler; (b) the initial aircraft spoiler Mises equivalent stress cloud chart; (c) the results of topology optimization.

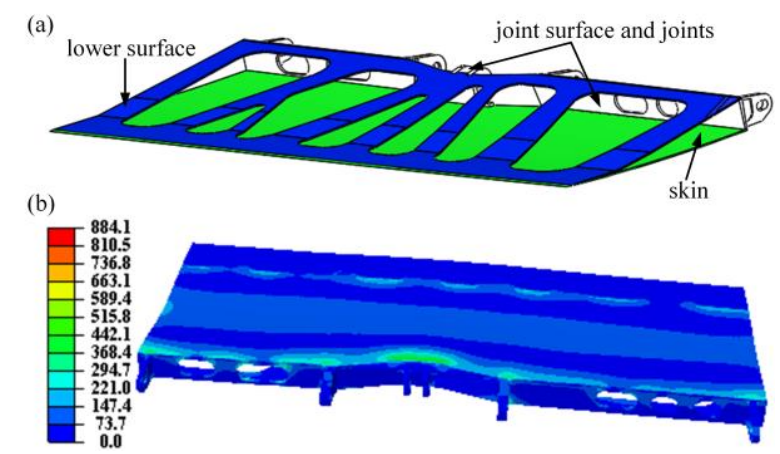

Figure 2. (a) The remodeled aircraft spoiler and (b) its stress distribution.

Figure 2(b) presents the corresponding stress distribution of the remodeled aircraft spoiler. The 
maximum stress is $884.1 \mathrm{MPa}$, which is easy to understand since we have removed the internal support. Subsequently, 3D kagome lattice structure is added to the inside of the aircraft spoiler. The rod diameter of each lattice unit is set as $5 \mathrm{~mm}$. The height of the 3D kagome lattice structure depends on the distance between the skin and the lower surface. Note that the positions of the lattice structures added are determined by the stress distributions presented in Figure 2(b); in other words, we employ the 3D kagome lattice structure to support the skins of the spoiler to reduce the stress concentration.

(a)

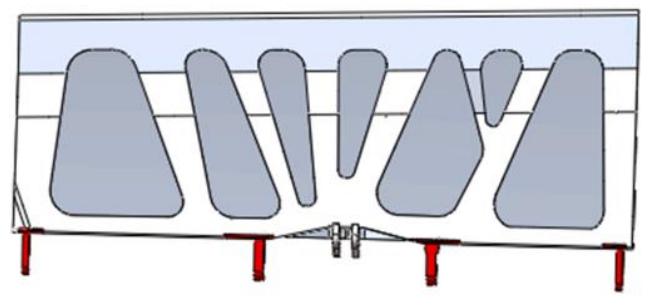

(b)

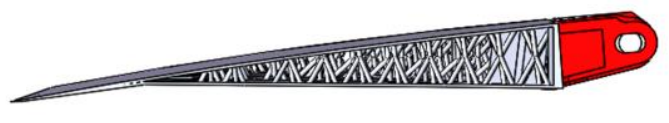

(c)

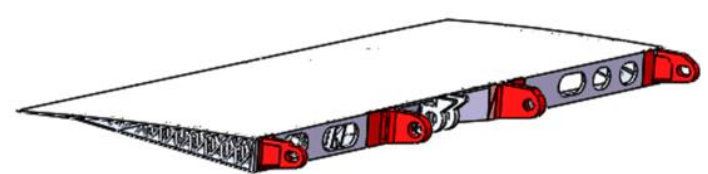

Figure 3. The designed aircraft spoiler: (a) top view, (b) lateral view, and (c)isometric view.

Figure 3(a)-(c) show the top view, the lateral view, and the isometric view of the designed aircraft spoiler, respectively. The stress concentration of the skin or the lower surface should be significantly reduced by adding lattice structures. However, the joints, which are non-designable can be regarded as support end of one cantilever, may exceed the maximum stress since we have added more materials to the skin or the lower surface by increasing their thickness. Therefore, Titanium alloy instead of the aluminum alloy are used to construct the joints. And in order to reduce the weight, the aluminum alloy is used for the other parts. 


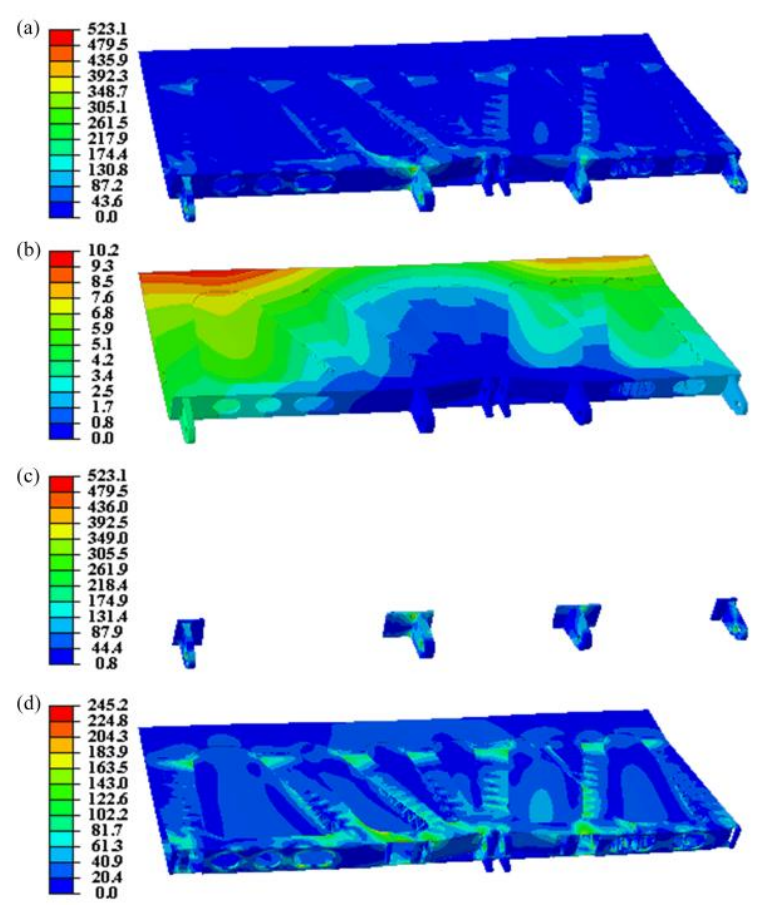

Figure 4. (a) The stress distributions of the designed aircraft spoiler; (b) the displacements of the designed aircraft spoiler; (c) the stress distributions of the joints 1, 2, 5, 6 and adjacent regions; (d) the stress distributions of other regions except for the joints 1,2, 5, 6 and adjacent regions.

Figure 4(a)-(b) present the corresponding stress and displacement distribution of the final design, showing that the maximum stress and the maximum displacement is $523.1 \mathrm{MPa}$ and $10.19 \mathrm{~mm}$, respectively. This small displacement means the high stiffness of the structure. In addition, the corresponding stress distributions of the regions with different material properties are also depicted in Figure 4(c)-(d). Specifically, the maximum stress of the joints 1, 2, 5, 6 and the adjacent regions is $523.1 \mathrm{MPa}$ (Figure 4c) and for other regions is 245.2 MPa. These two values are both smaller than the yield stress of the corresponding material used. The volume of the designed aircraft spoiler is $2.63 \times 10^{-3} \mathrm{~mm}^{3}$, and the weight is $7.772 \mathrm{~kg}$. Moreover, compared with the initial design structure (the joints and the skin use titanium alloy and the other parts use aluminum alloy) with a volume of $14.1 \times 10^{-3} \mathrm{~mm}^{3}$ and a weight of $40.395 \mathrm{~kg}$, the volume and weight of the designed aircraft spoiler are reduced by $81.35 \%$ and $80.76 \%$, respectively. Therefore, we have designed a novel aircraft spoiler with a high stiffness-to-weight ratio by using titanium alloy and aluminum alloy. Finally, considering the cost of 3D printing for metals, we fabricate the final designed aircraft spoiler with photosensitive resin by using a Jinshi high speed light curing 3D printer (Type: JS7255), as shown in Figure 5. 


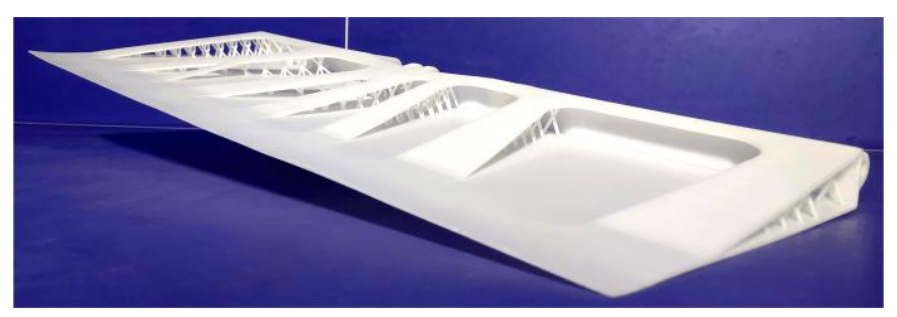

Figure 5. The real model for the designed aircraft spoiler.

It should be noted that the designed aircraft spoiler in this study is indeed not the best one since the empirical method is used and TO method is normally unable to obtain global optimum structure. Alternatively, TO method for lattice structures [46-49] may be another solution for the design of the aircraft spoiler. However, considering that the design is a large-scale problem, enormous amount of computing will be involved, especially if one wants to get the fine structures inside the aircraft spoiler [50].

\section{Conclusions}

We have designed a sandwich aircraft spoiler with a high stiffness-to-weight ratio by using two materials, i.e., titanium alloy and aluminum alloy. TO method are used to search for the best material distribution with maximizing the structural stiffness as the objective. The internal support materials obtained from the TO results are removed and subsequently replaced by 3D kagome lattice structures. To further improve the strength and at the same time reduce the weight of the aircraft spoiler, joints 1, 2, 5, 6, the key bearing components, use titanium alloy and the other parts use aluminum alloy. Results show that the volume and weight of the designed aircraft spoiler are reduced by $81.35 \%$ and $80.76 \%$, respectively, when compared with those of the initial design structure. In addition, the maximum stress and the maximum displacement of the final design is 523.1 MPa and $10.19 \mathrm{~mm}$, respectively, demonstrating that the designed aircraft spoiler can meet its service environment. We finally made a real model for the novel sandwich aircraft spoiler by using 3D printing.

\section{Acknowledgements:}

This research was financially supported by the Key Program of National Natural Science Foundation of China (No. 11832009). 


\section{References :}

[1] Wang, X. L., Wang, F. X., \& Li, Y. L. (2011). Aerodynamic characteristics of high-lift devices with downward deflection of spoiler. Journal of Aircraft, 48(2), 730-735

[2] Zhu, J. H., Zhang, W. H., \& Xia, L. (2016). Topology optimization in aircraft and aerospace structures design. Archives of Computational Methods in Engineering, 23(4), 595-622.

[3] Sigmund, O., \& Maute, K. (2013). Topology optimization approaches. Structural and Multidisciplinary Optimization, 48(6), 1031-1055.

[4] Huang, X., \& Xie, Y. M. (2010). A further review of ESO type methods for topology optimization. Structural and Multidisciplinary Optimization, 41(5), 671-683.

[5] Zhu, B., Chen, Q., Li, H., Zhang, H., \& Zhang, X. (2019). Design of planar large-deflection compliant mechanisms with decoupled multi-input-output using topology optimization. Journal of Mechanisms and Robotics, 1-11.

[6] Liu, J., \& Ma, Y. (2016). A survey of manufacturing oriented topology optimization methods. Advances in Engineering Software, 100, 161-175.

[7] Zhou, M., Fleury, R., Shyy, Y. K., Thomas, H., \& Brennan, J. (2002, September). Progress in topology optimization with manufacturing constraints. In 9th AIAA/ISSMO Symposium on Multidisciplinary Analysis and Optimization (p. 5614).

[8] Liu, J., Gaynor, A. T., Chen, S., Kang, Z., Suresh, K., Takezawa, A., ... \& Cheng, L. (2018). Current and future trends in topology optimization for additive manufacturing. Structural and Multidisciplinary Optimization, 1-27.

[9] Guo, X., Zhou, J., Zhang, W., Du, Z., Liu, C., \& Liu, Y. (2017). Self-supporting structure design in additive manufacturing through explicit topology optimization. Computer Methods in Applied Mechanics and Engineering, 323, 27-63.

[10] Zhang, K., Cheng, G., \& Xu, L. (2019). Topology optimization considering overhang constraint in additive manufacturing. Computers \& Structures, 212, 86-100.

[11] Mei, J., Liu, J., \& Liu, J. (2017). A novel fabrication method and mechanical behavior of all-composite tetrahedral truss core sandwich panel. Composites Part A: Applied Science and Manufacturing, 102, 28-39.

[12] Wei, K., Yang, Q., Ling, B., Xie, H., Qu, Z., \& Fang, D. (2018). Mechanical responses of titanium 3D kagome lattice structure manufactured by selective laser melting. Extreme Mechanics Letters, 23, 41-48.

[13] Liu, J., Chen, T., Zhang, Y., Wen, G., Qing, Q., Wang, H., .. \& Xie, Y. M. (2019). On sound insulation of pyramidal lattice sandwich structure. Composite Structures, 208, 385-394.

[14] Yin, H., Huang, X., Scarpa, F., Wen, G., Chen, Y., \& Zhang, C. (2018). In-plane crashworthiness of bio-inspired hierarchical honeycombs. Composite Structures, 192, 516-527.

[15] Remouchamps, A., Bruyneel, M., Fleury, C., \& Grihon, S. (2011). Application of a bi-level scheme including topology optimization to the design of an aircraft pylon. Structural and Multidisciplinary Optimization, 44(6), 739-750.

[16] Zhu, J. H., Gu, X. J., Zhang, W. H., \& Beckers, P. (2013). Structural design of aircraft skin stretch-forming die using topology optimization. Journal of Computational and Applied Mathematics, 246, 278-288. 
[17] Liu, S., Li, Q., Chen, W., Hu, R., \& Tong, L. (2015). H-DGTP—a Heaviside-function based directional growth topology parameterization for design optimization of stiffener layout and height of thin-walled structures. Structural and Multidisciplinary Optimization, 52(5), 903-913.

[18] Krog, L., Tucker, A., Kemp, M., \& Boyd, R. (2004, August). Topology optimisation of aircraft wing box ribs. In 10th AIAA/ISSMO multidisciplinary analysis and optimization conference (p. 4481).

[19] Maute, K. K., \& Reich, G. W. (2006). Integrated multidisciplinary topology optimization approach to adaptive wing design. Journal of Aircraft, 43(1), 253-263.

[20] Zhu, J., Zhang, W., \& Beckers, P. (2009). Integrated layout design of multi - component system. International journal for numerical methods in engineering, 78(6), 631-651.

[21] Xia, L., Zhu, J., \& Zhang, W. (2012). Sensitivity analysis with the modified Heaviside function for the optimal layout design of multi-component systems. Computer Methods in Applied Mechanics and Engineering, 241, 142-154.

[22] Yang, K., Zhu, J., Wu, M., \& Zhang, W. (2018). Integrated optimization of actuators and structural topology of piezoelectric composite structures for static shape control. Computer Methods in Applied Mechanics and Engineering, 334, 440-469.

[23] Roy, S., Crossley, W. A., Stanford, B., Moore, K. T., \& Gray, J. S. (2019). A Mixed Integer Efficient Global Optimization Algorithm with Multiple Infill Strategy-Applied to a Wing Topology Optimization Problem. In AIAA Scitech 2019 Forum (p. 2356).

[24] Dubois, A., Farhat, C., Abukhwejah, A. H., \& Shageer, H. M. (2018). Parameterization Framework for the MDAO of Wing Structural Layouts. AIAA Journal, 56(4), 1627-1638.

[25] Bhattacharyya, A., Conlan-Smith, C., \& James, K. A. (2019). Design of a Bi-stable Airfoil with Tailored Snap-through Response Using Topology Optimization. Computer-Aided Design, 108, 42-55.

[26] Guo, X., Zhang, W., \& Zhong, W. (2014). Doing topology optimization explicitly and geometrically - a new moving morphable components based framework. Journal of Applied Mechanics, 81(8), 081009.

[27] Zhang, W., Yuan, J., Zhang, J., \& Guo, X. (2016). A new topology optimization approach based on Moving Morphable Components (MMC) and the ersatz material model. Structural and Multidisciplinary Optimization, 53(6), 1243-1260.

[28] Zhang, W., Chen, J., Zhu, X., Zhou, J., Xue, D., Lei, X., \& Guo, X. (2017). Explicit three dimensional topology optimization via Moving Morphable Void (MMV) approach. Computer Methods in Applied Mechanics and Engineering, 322, 590-614.

[29] Guo, X., Zhang, W., Zhang, J., \& Yuan, J. (2016). Explicit structural topology optimization based on moving morphable components (MMC) with curved skeletons. Computer methods in applied mechanics and engineering, 310, 711-748.

[30] Costa Jr, J. C. A., \& Alves, M. K. (2003). Layout optimization with h - adaptivity of structures. International Journal for Numerical Methods in Engineering, 58(1), 83-102.

[31] Stainko, R. (2006). An adaptive multilevel approach to the minimal compliance problem in topology optimization. Communications in numerical methods in engineering, 22(2), 109-118.

[32] Wang, Y., Kang, Z., \& He, Q. (2013). An adaptive refinement approach for topology optimization based on separated density field description. Computers \& Structures, 117, 
$10-22$.

[33] Wang, Y., Kang, Z., \& He, Q. (2014). Adaptive topology optimization with independent error control for separated displacement and density fields. Computers \& Structures, 135, 50-61.

[34] Wang, H., Liu, J., \& Wen, G. (2019). An efficient evolutionary structural optimization method with smooth edges based on the game of building blocks. Engineering Optimization, doi: https://doi.org/10.1080/0305215X.2018.1562550.

[35] Bruggi, M., \& Verani, M. (2011). A fully adaptive topology optimization algorithm with goal-oriented error control. Computers \& Structures, 89(15-16), 1481-1493.

[36] Wang, H., Liu, J., \& Wen, G. An adaptive mesh - adjustment strategy for continuum topology optimization to achieve manufacturable structural layout. International Journal for Numerical Methods in Engineering, doi: https://doi.org/10.1002/nme.6001.

[37] Xia, Q., Shi, T., \& Xia, L. (2019). Stable hole nucleation in level set based topology optimization by using the material removal scheme of BESO. Computer Methods in Applied Mechanics and Engineering, 343, 438-452.

[38] Zhu, B., Zhang, X., Liu, M., Chen, Q., \& Li, H. (2019). Topological and Shape Optimization of Flexure Hinges for Designing Compliant Mechanisms Using the Level Set Method. Chinese Journal of Mechanical Engineering, 32(1), 13.

[39] Sigmund, O. (2001). A 99 line topology optimization code written in Matlab. Structural and multidisciplinary optimization, 21(2), 120-127.

[40] Svanberg, K. (1987). The method of moving asymptotes - a new method for structural optimization. International journal for numerical methods in engineering, 24(2), 359-373.

[41] Zuo, W., \& Saitou, K. (2017). Multi-material topology optimization using ordered SIMP interpolation. Structural and Multidisciplinary Optimization, 55(2), 477-491.

[42] Rong, J. H., Tang, Z. L., Xie, Y. M., \& Li, F. Y. (2013). Topological optimization design of structures under random excitations using SQP method. Engineering Structures, 56, 2098-2106.

[43] Long, K., Wang, X., \& Liu, H. (2018). Stress-constrained topology optimization of continuum structures subjected to harmonic force excitation using sequential quadratic programming. Structural and Multidisciplinary Optimization, 1-13.

[44] Dunning, P. D., \& Kim, H. A. (2015). Introducing the sequential linear programming level-set method for topology optimization. Structural and Multidisciplinary Optimization, 51(3), 631-643.

[45] Liu, J., Wen, G., \& Xie, Y. M. (2016). Layout optimization of continuum structures considering the probabilistic and fuzzy directional uncertainty of applied loads based on the cloud model. Structural and Multidisciplinary Optimization, 53(1), 81-100.

[46] Hu, Z., Gadipudi, V. K., \& Salem, D. R. (2019). Topology Optimization of Lightweight Lattice Structural Composites Inspired by Cuttlefish Bone. Applied Composite Materials, 26(1), 15-27.

[47] Xiao, Z., Yang, Y., Xiao, R., Bai, Y., Song, C., \& Wang, D. (2018). Evaluation of topology-optimized lattice structures manufactured via selective laser melting. Materials \& Design, 143, 27-37.

[48] Wu, Z., Xia, L., Wang, S., \& Shi, T. (2019). Topology optimization of hierarchical lattice structures with substructuring. Computer Methods in Applied Mechanics and Engineering, 345, 602-617. 
[49] Song, J., Wang, Y., Zhou, W., Fan, R., Yu, B., Lu, Y., \& Li, L. (2019). Topology optimization-guided lattice composites and their mechanical characterizations. Composites Part B: Engineering, 160, 402-411.

[50] Aage, N., Andreassen, E., Lazarov, B. S., \& Sigmund, O. (2017). Giga-voxel computational morphogenesis for structural design. Nature, 550(7674), 84. 